\title{
Risk factors and outcomes for acute respiratory failure in coronavirus disease 2019: An observational cohort study
}

\author{
Paweł Piwowarczyk ${ }^{1, A-F}$, Marta Szczukockad,B-F, Paweł Kutnik2,B-F , Michał Borys, ${ }^{1, B-F}$, \\ Anna Mikłaszewska ${ }^{3, B-F}$, Sławomir Kiciak ${ }^{3, B-F}$, Mirosław Czuczwar, ${ }^{1, B-F}$ \\ ${ }^{1}$ Department of Anaesthesiology and Intensive Therapy, Medical University of Lublin, Poland \\ 2 Student's Scientific Association at the Department of Anaesthesiology and Intensive Therapy, Medical University of Lublin, Poland \\ ${ }^{3}$ Department of Infectious Diseases, Medical University of Lublin, Poland \\ A - research concept and design; $\mathrm{B}$ - collection and/or assembly of data; C - data analysis and interpretation; \\ $D$ - writing the article; $E$ - critical revision of the article; $F$ - final approval of the article
}

Address for correspondence

Paweł Piwowarczyk

E-mail: piwowarczyk.pawel@gmail.com

Funding sources

None declared

Conflict of interest

None declared

Received on July 8, 2020

Reviewed on 0ctober 25, 2020

Accepted on November 18, 2020

Published online on December 31, 2020

\section{Abstract}

Background. The novel severe acute respiratory syndrome coronavirus 2 (SARS-CoV-2), has spread throughout Europe. However, there is a lack of data on the full clinical course of patients infected with SARS-CoV-2 in Europe, especially in the population that developed acute respiratory failure (ARF).

Objectives. To identify risk factors associated with developing ARF during SARS-CoV-2 infection.

Materials and methods. This was an observational study of 60 adult patients with laboratory-confirmed SARS-CoV-2 infection. Data were collected from March 26, 2020 to May 26, 2020 in a tertiary academic hospital in Poland. All patients reached final outcome (discharge from the hospital or death). We divided patients into 2 groups based on whether they developed ARF, compared their clinical data, and performed multivariate logistic regression.

Results. Twenty-two patients (36\%) from the observed cohort developed ARF. Logistic regression identified that a high sequential organ failure assessment score at admission (odds ratio $(O R)=6.97(1.57-30.90$, $p=0.011)$ ), and a long time from admission until pneumonia $(O R=1.41(1.06-1.87, p=0.016))$, correlated with ARF development. D-dimer, lactate dehydrogenase, neutrophil to lymphocyte ratio, (-reactive protein (CRP), and interleukin 6 (IL-6) differed both statistically and clinically between ARF and non-ARF groups. The mortality rate in the observed cohort of patients was 13.3\%, and it was $32 \%$ in the group that developed ARF.

Conclusions. Routine vigilant examination of the above markers may identify patients at the highest risk of ARF early on during COVID-19 infection.

Key words: coronavirus, clinical characteristics, observational, SARS, respiratory failure

Cite as

Piwowarczyk P, Szczukocka M, Kutnik P, et al. Risk factors and outcomes for acute respiratory failure in coronavirus disease 2019: An observational cohort study. Adv Clin Exp Med. 2021;30(2):165-171. doi:10.17219/acem/130603

DOI

10.17219/acem/130603

Copyright

Copyright by Author(s)

This is an article distributed under the terms of the

Creative Commons Attribution 3.0 Unported (CC BY 3.0)

(https://creativecommons.org/licenses/by/3.0/) 


\section{Background}

During the past 2 decades, 2 major epidemics were caused by viruses belonging to the betacoronavirus group: severe acute respiratory syndrome coronavirus (SARS$\mathrm{CoV}$ ) in 2002 and the Middle East respiratory syndrome coronavirus (MERS-CoV) in 2012. ${ }^{1,2}$ In December 2019, China reported the outbreak of pneumonia of an unknown origin in Wuhan, the capital of Hubei province. ${ }^{3}$ The pathogen responsible for the outbreak was identified as a novel coronavirus (SARS-CoV-2) belonging to the betacoronavirus group and causing COVID-19 infection. On March 12, 2020, the World Health Organization (WHO) announced a COVID-19 pandemic, which has resulted to date in 24.48 million confirmed cases of COVID-19 worldwide and 832.378 causalities (data as of August 28, 2020). In Poland, there have been 65,480 confirmed cases, causing 2,018 deaths (data as of August 28, 2020). ${ }^{4}$

The clinical manifestations of COVID-19 include fever, dry cough, dyspnea, fatigue, a normal or decreased leucocyte count, and specific findings in chest imaging, including $\mathrm{X}$-ray, computed tomography (CT) scan, and ultrasound examination. ${ }^{5,6} \mathrm{~A}$ small proportion of patients presents symptoms of acute respiratory failure (ARF) or acute respiratory distress syndrome (ARDS), requiring the implementation of more advanced ventilatory support and subsequent admission to the intensive care unit (ICU). Accumulating data shows that ICU admission is associated with poor prognosis with a range of mortality from $30.9 \%^{6}$ to $81.3 \%$ in the cohort of ICU patients treated in the New York City area. ${ }^{7}$ A recent meta-analysis reports that mortality rates in patients with COVID-19 treated in ICU have fallen to $40 \%$, which is higher than in other types of viral pneumonia. ${ }^{8}$ Exploring the association of patients' outcomes with the clinical presentation of the disease is urgently needed, especially in the face of rapidly changing phenotype of the virus in particular populations. Moreover, the mortality rates reported in the majority of retrospective studies may present a skewed picture since a large proportion of critically ill patients are still treated in the ICU and did not reach the outcome.

\section{Objectives}

This study aimed to provide a detailed description of patients' characteristics, treatment modalities and outcomes in the Polish cohort treated due to COVID-19 at a tertiary academic hospital.

\section{Materials and methods}

This observational cohort study was approved by the Ethical Committee of the Medical University of Lublin, Poland (approval No. KE-0254/59/2020). All the study data was collected in a tertiary academic hospital in Lublin, Poland.
We included adult patients hospitalized in the Department of Anesthesiology and Intensive Care and the Department of Infectious Diseases. The study adhered to the STROBE (STrengthening the Reporting of Observational studies in Epidemiology) guidelines for collecting and interpreting observational data. All analyzed patients had a confirmed SARS-CoV-2 infection with real-time reverse transcription polymerase chain reaction (RT-PCR). Oral consent was obtained from each conscious patient. In the case of 3 patients who were transferred to our hospital from regional ICUs, guardianship court consent was obtained. All included patients were followed until the outcome (discharge from the hospital or death) was reached. Patients suspected with COVID-19 infection but unconfirmed with RT-PCR and individuals who did not give consent to participate in the study or withdrew consent were excluded from the study.

The main goal of this study was to identify the factors and outcomes associated with the development of ARF in the population of patients with confirmed COVID-19. All observed patients were divided into 2 groups (ARF and non-ARF groups) according to the results of arterial blood gas analysis and the extent of oxygenation disorders. The most recent definition of ARDS, the Berlin definition, ARDS is defined by a combination of acute hypoxemia $\left(\mathrm{PaO}_{2} / \mathrm{FiO}_{2} \leq 300 \mathrm{~mm} \mathrm{Hg}\right)$, in a ventilated patient with a positive end-expiratory pressure (PEEP) of at least $5 \mathrm{~cm}$ $\mathrm{H}_{2} \mathrm{O}$, and bilateral opacities not fully explained by heart failure or volume overload. Patients included in the ARF group have fulfilled the $1^{\text {st }}$ criterion of ARDS that concerns the extent of oxygenation disorders. However, not all of them have completed the $2^{\text {nd }}$ and $3^{\text {rd }}$ criteria concerning bilateral chest radiological changes and the presence of positive expiratory pressure of at least $5 \mathrm{~cm}$ of water. We did not use non-invasive ventilation due to a lack of safe interfaces for its provision and the associated threat to the staff. The secondary goal of the study was a comparison of baseline demographics, epidemiological data (history of an infected family member, history of traveling abroad in the past 2 weeks), symptoms at admission (fever, dry cough, hemoptysis, myalgia, headache, diarrhea, dyspnea), laboratory results at admission (white blood count (WBC), lymphocytes, neutrophils, neutrophil to lymphocyte ratio (NLR), immature granulocytes, platelets, hemoglobin, D-dimers, prothrombin time, activated partial thromboplastin time, aspartate transaminase (AST), alanine transaminase (ALT), as well as serum levels of creatinine, urea, bilirubin, lactate dehydrogenase (LDH), procalcitonin, C-reactive protein (CRP) and interleukin 6 (IL-6). We calculated the sequential organ failure assessment (SOFA) score at admission to the hospital and initiated monitoring of hemoglobin oxygen saturation in arterial blood $\left(\mathrm{SpO}_{2}\right)$. We obtained an arterial blood sample in patients presenting with $\mathrm{SpO}_{2}$ below 94\% or in need of oxygen supplementation; subsequently, we calculated the arterial oxygen partial pressure $\left(\mathrm{PaO}_{2} / \mathrm{FiO}_{2}\right)$ ratio. We evaluated pharmacotherapy and organ support 
therapies in the observed cohort, and we recorded and compared baseline chest X-rays. The data were collected from March 26, 2020 to May 26, 2020, with the latter being the day of discharge of the last included patient. All patients requiring invasive mechanical ventilation, veno-venous extracorporeal membrane oxygenation (V-V ECMO), continuous renal replacement therapy, or administration of catecholamines were treated in the ICU, whereas patients requiring pharmacotherapy or oxygen supplementation only were treated in the infectious diseases ward.

Categorical variables were presented as frequency rates and percentages. Continuous variables were tested for normal distribution using the Kolmogorov-Smirnov test; they were presented as means and 95\% confidence intervals (95\% CIs) for normally distributed data and as medians and interquartile range (IQR 1-3) for non-normally distributed data. Logistic regression was presented as an odds ratio (OR) with 95\% CI. The sensitivity of the calculated model was presented as the area under the curve (AUC) of a receiver operating characteristic (ROC) curve. All statistical calculations were performed using STATISTICA v. 13.3 (StatSoft Inc., Tulsa, USA). A p-value of 0.05 or below was considered statistically significant.

\section{Results}

The study population included 60 patients with a laboratory-confirmed presence of SARS-CoV-2 RNA. The demographic, clinical, and epidemiological characteristics of all included patients are presented in Table 1. The laboratory variables at the time of admission to the hospital in both groups are compared in Table 2. The median time from the first symptoms to the diagnosis of pneumonia was 7 days (4-8 days). The clinical course of 22 patients (36\%) deteriorated during hospitalization because of the development of ARF. Ten patients (16.7\%) were intubated and mechanically ventilated. We initiated $\mathrm{V}-\mathrm{V}$ ECMO in 3 patients (2\%). The number of patients who needed extracorporeal organ support and infusion

Table 1. Demographic, clinical, and epidemiological characteristics of patients. Data are presented as numbers and percentages for categorical values and as median and IQR for continuous values. Statistical analysis was performed accordingly

\begin{tabular}{|c|c|c|c|}
\hline Parameter & $\operatorname{ARF}(n=22)$ & Non-ARF $(n=38)$ & p-value \\
\hline \multicolumn{4}{|c|}{ Demographics, n (\%) } \\
\hline Male sex & $16(73 \%)$ & $15(39 \%)$ & $0.013^{*}$ \\
\hline Female sex & $6(27 \%)$ & $23(61 \%)$ & 0.013 \\
\hline Age, [years] (IQR) & $59(50-67)$ & $39(25-51)$ & $0.00006^{*}$ \\
\hline $18-29$ & $1(4.5 \%)$ & $12(30.8 \%)$ & 0.014 \\
\hline $30-44$ & $2(9.1 \%)$ & $14(36.8 \%)$ & 0.019 \\
\hline $45-65$ & $9(40.9 \%)$ & $10(26.3 \%)$ & 0.24 \\
\hline $65+$ & $9(40.9 \%)$ & $2(5.3 \%)$ & $0.0006^{*}$ \\
\hline \multicolumn{4}{|c|}{ Symptoms, n (\%) } \\
\hline Fever & $21(95.5 \%)$ & $21(55.3 \%)$ & $0.001^{*}$ \\
\hline Cough & $20(90.9 \%)$ & $22(57.9 \%)$ & $0.009^{*}$ \\
\hline Muscle ache & $5(22.7 \%)$ & $13(34.2 \%)$ & 0.32 \\
\hline Productive cough & $5(22.7 \%)$ & $4(10.5 \%)$ & 0.22 \\
\hline Headaches & $5(22.7 \%)$ & $4(10.5 \%)$ & 0.22 \\
\hline Hemoptysis & $2(9.1 \%)$ & $0(0 \%)$ & 0.135 \\
\hline Diarrhea & $7(31.8 \%)$ & $9(23.6 \%)$ & 0.53 \\
\hline \multicolumn{4}{|c|}{ Comorbidities, n (\%) } \\
\hline COPD & $1(4.5 \%)$ & $(0 \%)$ & 0.37 \\
\hline Liver disease & $2(9.1 \%)$ & $(0 \%)$ & 0.13 \\
\hline Diabetes & $2(9.1 \%)$ & $2(5.3 \%)$ & 0.56 \\
\hline Chronic kidney disease & $(0 \%)$ & $(0 \%)$ & N.A. \\
\hline Hypertension & $12(54.5 \%)$ & $8(21.1 \%)$ & $0.008^{*}$ \\
\hline Cardiovascular disease & $6(27.3 \%)$ & $2(5.3 \%)$ & $0.016^{*}$ \\
\hline \multicolumn{4}{|c|}{ Epidemiology } \\
\hline Confirmed SARS-COV-2 in family & $12(54.5 \%)$ & $25(65.8 \%)$ & 0.32 \\
\hline Traveling abroad within 2 weeks prior to confirmation of SARS-COV-2 & $2(9.1 \%)$ & $4(10.5 \%)$ & 0.83 \\
\hline
\end{tabular}

ARF - acute respiratory failure; IQR - interquartile range; SARS-CoV-2 - severe acute respiratory syndrome coronavirus 2; COPD - chronic obstructive pulmonary disease; ${ }^{*} p$ values indicate differences between ARF and non-ARF patients; $p<0.05$ was considered statistically significant. 
Table 2. Laboratory and chest radiogram findings. Data are presented as numbers and percentages for categorical values and as median and IQR for continuous values. Statistical analysis was performed accordingly

\begin{tabular}{|c|c|c|c|c|}
\hline Parameter & Norms & $\operatorname{ARF}(n=22)$ & Non-ARF $(n=38)$ & $p$-value \\
\hline WBC [K/uL] & $4-10$ & 6.49 [5.39-9.84] & $4.99[4.18-5.79]$ & 0.0002 \\
\hline Lymphocytes [K/uL] & $0.8-4.3$ & $1.02[0.62-1.31]$ & $1.65[1.22-2.18]$ & 0.0004 \\
\hline Neutrophils [K/uL] & $2.25-7$ & $5.55[4.1-8.42]$ & 2.66 [1.94-3.28] & 0.00004 \\
\hline NLR & $1-3$ & $8.53[5.32-13.46]$ & $2.96[2.11-4.39]$ & $0.000003^{*}$ \\
\hline IG [K/uL] & $0-0.25$ & $0.06[0.03-0.12]$ & $0.01[0.01-0.03]$ & 0.000002 \\
\hline Hemoglobin [g/dL] & $12-16$ & $13.5[12.7-14.7]$ & 14 [13.3-15.6] & 0.08 \\
\hline Platelets [K/uL] & $130-400$ & 167 [140-209] & 199 [163-239] & 0.16 \\
\hline $\mathrm{PT}[\mathrm{s}]$ & $11-14$ & $13.6[12.5-15.5]$ & 12.5 [12.2-13.3] & 0.01 \\
\hline APTT [s] & $28-38$ & 31.8 [30.4-39.3] & $34.2[29.5-36.7]$ & 0.26 \\
\hline D-dimers [ng/mL] & $<500$ & 758 [450-1900] & 418 [309-773] & $0.02^{*}$ \\
\hline Bilirubin [mg/dL] & $0.2-11$ & $0.43[0.27-0.52]$ & $0.39[0.24-0.65]$ & 0.88 \\
\hline ALAT $[\mathrm{U} / \mathrm{L}]$ & $5-34$ & 31.3 [23.7-40.8] & 19.9 [13.4-27.8] & 0.004 \\
\hline ASPAT [IU/L] & $5-35$ & $35.0[23.5-56]$ & 20.7 [15.4-25] & 0.0006 \\
\hline LDH [IU/L] & $10-480$ & $633[526-826]$ & 355.5 [287-412.5] & 0.00003 \\
\hline Creatinine $[\mathrm{mg} / \mathrm{dL}]$ & $0.5-1.2$ & $1.02[0.80-1.27]$ & $0.93[0.71-1.02]$ & 0.013 \\
\hline Urea [mg/dL] & $15-40$ & $32.5[26.7-47.4]$ & $28.1[24.1-34.7]$ & 0.018 \\
\hline IL-6 [pg/mL] & $5-15$ & 61 [8.87-111.5] & 7.6 [2.67-18.83] & 0.006 \\
\hline PCT $[\mathrm{ng} / \mathrm{mL}]$ & $<2$ & $0.14[0.085-0.25]$ & $0.05[0.04-0.07]$ & 0.0001 \\
\hline CRP [mg/L] & $<5$ & $69.2[29.2-206.7]$ & $2.7[1.3-8.2]$ & $0.00000^{*}$ \\
\hline \multicolumn{5}{|c|}{ Chest radiogram quadrants } \\
\hline 0 & & $3(13.6 \%)$ & $34(89.5 \%)$ & $0.002^{*}$ \\
\hline 1 & & $2(9.1 \%)$ & $4(10.5 \%)$ & 1 \\
\hline 2 & & $10(45.4 \%)$ & 0 & $0.002^{*}$ \\
\hline 3 & & $2(9.1 \%)$ & 0 & 0.15 \\
\hline 4 & & $5(22.7 \%)$ & 0 & $0.01^{*}$ \\
\hline
\end{tabular}

IQR - interquartile range; WBC - white blood count; NLR - neutrophil to lymphocyte ratio; IG - immature granulocytes (\%); PT - prothrombin time; APTT - activated partial thromboplastin time; ALAT - alanine transaminase; ASPAT - aspartate transaminase; LDH - lactate dehydrogenase; IL-6 - interleukin 6; PCT - procalcitonin; CRP - C-reactive protein; ${ }^{*} \mathrm{p}$-values indicate statistical and clinical differences between ARF and non-ARF patients; $\mathrm{p}<0.05$ was considered statistically significant.

of vasoactive and inotropic agents is presented in Table 3. Eight patients (13.3\%) died in the entire cohort. The survival rate was $68 \%(15 / 22)$ in the ARF group and $97 \%$ (37/38) in the non-ARF group. One patient was readmitted 16 days after the initial mild course of COVID-19. While the SARS-CoV-2 RNA test was negative during the $2^{\text {nd }}$ admission, the patient died because of lung fibrosis.

We performed an additional sub-analysis in the population of COVID-19 patients who developed ARF. The baseline characteristics and treatment modalities were compared between the groups of survivors and non-survivors. Interestingly, the SOFA score at admission did not differ between the groups of ARF survivors and non-survivors (2 vs $5 ; \mathrm{p}=0.09$ ). The extent of oxygenation disorders was similar in both groups. The need for mechanical ventilation and norepinephrine support that occurred in $45 \%$ and $45.7 \%$ of the patients from the ARF group, respectively, was associated with a negative outcome.
Our multivariate logistic regression analysis detected 2 variables associated with ARF development: $\mathrm{OR}=6.97$ $(1.57-30.9, \mathrm{p}=0.011)$ and $\mathrm{OR}=1.41(1.06-1.87, \mathrm{p}=0.016)$, respectively.

\section{Discussion}

All patients included in this observational trial reached the outcome (death or discharge from the hospital). The data presented in our study highlighted several factors associated with the development of ARF in the patients treated in our hospital. We found that the main elements correlating with a severe course of COVID-19 are demographics, comorbidities and various laboratory findings. In our study, male gender and advanced age were found to be associated with a higher risk of developing ARF. The association between advanced age and a more severe course of SARS CoV-2 infection was previously 
Table 3. Pharmacotherapy, organ support and outcome. Data are presented as numbers and percentages

\begin{tabular}{|c|c|c|c|}
\hline Variable & $\operatorname{ARF}(n=22)$ & Non-ARF $(n=38)$ & $\mathrm{p}$-value \\
\hline \multicolumn{4}{|c|}{ Pharmacotherapy } \\
\hline Ritonavir - lopinavir & $17(77.3 \%)$ & $13(34.2 \%)$ & $0.0017^{*}$ \\
\hline Antibiotics & $21(95.5 \%)$ & $12(31.6 \%)$ & $<0.0001^{*}$ \\
\hline Chloroquine & $15(68.2 \%)$ & $15(39.5 \%)$ & $0.04^{*}$ \\
\hline Steroids & $8(36.7 \%)$ & $0(0 \%)$ & $0.0001^{*}$ \\
\hline \multicolumn{4}{|c|}{ Organ support and survival } \\
\hline Oxygen supplementation & $22(100 \%)$ & $0(0 \%)$ & \\
\hline Invasive ventilation & $10(45 \%)$ & 0 & N/A \\
\hline ECMO & $3(13 \%)$ & 0 & N/A \\
\hline Dobutamine infusion & $4(18.1 \%)$ & 0 & $\mathrm{~N} / \mathrm{A}$ \\
\hline Norepinephrine infusion & $10(45.4 \%)$ & 0 & N/A \\
\hline CRRT & $5(22 \%)$ & 0 & N/A \\
\hline Survival & $15(68 \%)$ & 37 (97\%) & 0.0013 \\
\hline
\end{tabular}

ECMO - extracorporeal membrane oxygenation; CRRT - continuous renal replacement therapy; * $p$-values indicate differences between ARF and non-ARF patients; $p<0.05$ was considered statistically significant.

reported. ${ }^{9,10}$ We found cough and fever to be the dominant symptoms at admission to the hospital. This finding is in line with previously published results showing that both symptoms are observed in $88.7 \%$ and $57.6 \%$ of COVID-19 patients, respectively. ${ }^{11}$ Moreover, we identified a significantly higher prevalence of cough and fever in the ARF group (Table 1). Muscle ache, productive cough, headaches, hemoptysis, and diarrhea were not associated with ARF occurrence, contrary to hemoptysis, which was only observed in the ARF group (Table 1).

Arterial hypertension and cardiovascular disease (CVD) were found to be risk factors for an adverse outcome in multiple retrospective COVID-19 studies. ${ }^{12,13}$ Similarly, we found that the population of patients with hypertension or CVD was at an increased risk of developing ARF. However, it is noteworthy that we did not observe any correlation between the use of antihypertensive drugs and COVID-19 severity, which is in line with the recently published results from a large retrospective study in the USA (Table 1). ${ }^{14}$ Moreover, the presence of other comorbidities was not associated with the development of ARF. It should be noted, however, that the number of patients suffering from these diseases in our study was relatively small. In our study, $19 / 22$ patients (86.4\%) in the ARF group and 4/38 patients $(10.5 \%)$ in the non-ARF group developed radiological signs of pneumonia in the chest X-ray. We found that a long time from the first presentation of symptoms to radiological signs of pneumonia correlates with a more severe course of COVID-19, which is consistent with the findings of previously published reports..$^{15}$

We found that many laboratory results correlate with the development of ARF. Although the majority of different variables were within the laboratory norm, we found discrepancies in the NLR, lactate dehydrogenase, D-dimers, CRP, and IL-6, which were statistically significant and potentially clinically useful. A high NLR in COVID-19 patients was previously recognized as a useful predictive marker with $88 \%$ sensitivity, $63.6 \%$ specificity, and a calculated AUC of $0.841{ }^{16}$ We observed significantly higher $\mathrm{LDH}$ levels in the ARF group, which is consistent with previous findings showing that elevated LDH levels are associated with a more severe course of COVID-19.6,17 We identified a significant correlation between elevated Ddimer levels and an increased risk of ARF, which is in line with the findings of previously conducted cohort studies. ${ }^{18}$ Interestingly, a fourfold increase in D-dimer levels $(>2.0 \mu \mathrm{g} / \mathrm{mL})$ predicted in-hospital mortality with a sensitivity of $92.3 \%$ and a specificity of $83.3 \%$, which makes this parameter a potentially useful tool for a negative prognosis. ${ }^{19}$ Two analyzed pro-inflammatory markers, IL- 6 and CRP, positively correlated with an increased risk of ARF. This observation is consistent with previously reported data on the potential role of both IL- 6 and CRP in the early identification of patients at a high risk of a severe course of COVID-19. ${ }^{20}$ According to the literature, CRP is suggested to have a positive correlation not only with the severity of presentation in SARS-CoV-2 infection but also with the diameter of lung lesions and negative outcome. ${ }^{21,22}$

In this study, most patients who developed ARF because of SARS-Cov-2 infection presented alterations in their chest X-rays. These included interstitial infiltrates or ground-glass opacities in at least 2 lung quadrants. Interestingly, none of the patients in the non-ARF group showed changes in their chest X-ray exceeding 1 quadrant of the lung. These findings could support the use of chest X-ray to facilitate diagnosis and predict the severity of the disease, especially when a chest CT scan is unavailable or not feasible. It should be noted that most authors challenge the clinical utility and validity of chest 
Table 4. Pharmacotherapy, organ support and outcome in the sub-group of ARF patients. Data is presented as numbers and percentages

\begin{tabular}{|c|c|c|c|}
\hline Variable & ARF survivors $(n=15)$ & ARF non-survivors $(n=7)$ & $\mathrm{p}$-value \\
\hline SOFA & $2[2-4]$ & $5[3-7]$ & 0.09 \\
\hline \multicolumn{4}{|c|}{$\mathrm{PaO}_{2} / \mathrm{FiO}_{2}$} \\
\hline $200-300$ & $8(53.3 \%)$ & $1(14.3 \%)$ & 0.08 \\
\hline $100-200$ & $5(33.3 \%)$ & $3(43.9 \%)$ & 0.66 \\
\hline$<100$ & $2(13.3 \%)$ & $3(43.9 \%)$ & 0.12 \\
\hline \multicolumn{4}{|c|}{ Pharmacotherapy } \\
\hline Ritonavir/lopinavir & $14(93.3 \%)$ & $3(43.9 \%)$ & $0.0085^{*}$ \\
\hline Antibiotics & $14(93.3 \%)$ & $7(100 \%)$ & 1 \\
\hline Chloroquine & $13(86.7 \%)$ & $2(28.6 \%)$ & $0.0064^{*}$ \\
\hline Steroids & $4(26.6 \%)$ & $4(57.1 \%)$ & 0.17 \\
\hline \multicolumn{4}{|c|}{ Organ support } \\
\hline Oxygen supplementation & $15(100 \%)$ & $7(100 \%)$ & 1.0 \\
\hline Invasive ventilation & $3(20 \%)$ & $6(85.7 \%)$ & $0.004^{*}$ \\
\hline ECMO & $2(13.3 \%)$ & $1(14.3 \%)$ & 0.95 \\
\hline Dobutamine infusion & $2(13.3 \%)$ & $2(28.5 \%)$ & 0.39 \\
\hline Norepinephrine infusion & $4(26.6 \%)$ & $6(85.7 \%)$ & $0.0096^{*}$ \\
\hline CRRT & $4(26.6 \%)$ & $1(14.2 \%)$ & 0.52 \\
\hline
\end{tabular}

SOFA - sequential organ failure assessment; ECMO - extracorporeal membrane oxygenation; CRRT - continuous renal replacement therapy; ${ }^{*} \mathrm{p}$-values indicate differences between ARF survivors and non-survivors; $p<0.05$ was considered statistically significant.

X-ray in patients with COVID-19. The rationale is a reported high prevalence of negative chest X-ray examinations in up to $60 \%$ of cases, which was not observed in our study. $^{23}$

We evaluated the pharmacotherapy administered in both study groups and found that steroids, ritonavir/ lopinavir, antibiotics, and chloroquine were significantly more frequently used in the group that developed ARF (Table 2). Our results on the pharmacotherapy used correspond with those presented by Wang et al., which were reported from Wuhan, China, where significantly more groups of drugs were used in patients who deteriorated and were transferred to the ICU. ${ }^{5}$ We could not evaluate the impact of pharmacotherapy on outcomes. However, recent evidence report that the use of dexamethasone in patients requiring oxygen supplementation or mechanical ventilation reduces 28 -day mortality. ${ }^{24}$ Remdesivir is another therapeutic option reported to significantly shorten the recovery time but not affect the mortality. ${ }^{25}$

Our sub-analysis performed in the group that developed ARF from the observed cohort showed that the need for invasive mechanical ventilation and norepinephrine infusion was associated with increased mortality (Table 4). The low survival rate in the population receiving advanced respiratory support (33\%) observed in our study is identical to that reported in the British National Intensive Care Audit on the outcomes of COVID-19 treatment $(33.8 \%){ }^{26}$

\section{Limitations}

This study has several limitations. Because of the observational nature of the study and its relatively small cohort size, the results are exposed to treatment and selection bias. The causal effect of the associations drawn cannot be determined. Furthermore, many examinations, including routine CT chest scanning at admission, could not be performed because of institutional limitations. Data regarding the pharmacotherapy used and any potential association with the outcome should be taken with caution.

\section{Conclusions}

This observational trial provides information on the demographic, clinical and epidemiological features as well as outcomes of 60 patients diagnosed with COVID-19 treated in a tertiary center in Poland. The risk factors associated with the development of ARF in 37\% of the studied patients included hypertension, CVD, advanced age, male gender, high SOFA score, and a long time from the occurrence of symptoms until pneumonia. Routine D-dimer, LDH, NLR, CRP, and IL-6 examination may have the potential to identify patients at the highest risk of developing ARF during COVID-19. The mortality rate in the observed cohort of patients was $13.3 \%$, and it was $32 \%$ in the group that developed ARF. 


\section{ORCID iDs}

Paweł Piwowarczyk (D) https://orcid.org/0000-0002-8529-484X Marta Szczukocka (D) https://orcid.org/0000-0003-1431-5495 Paweł Kutnik (D) https://orcid.org/0000-0002-8434-6812 Michał Borys (D) https://orcid.org/0000-0002-6183-811X Anna Mikłaszewska (D) https://orcid.org/0000-0002-8666-2202 Sławomir Kiciak (D) https://orcid.org/0000-0002-4031-0076 Mirosław Czuczwar (D) https://orcid.org/0000-0002-9025-6717

\section{References}

1. Kuiken T, Fouchier RA, Schutten M, et al. Newly discovered coronavirus as the primary cause of severe acute respiratory syndrome. Lancet. 2003;362(9380):263-270.

2. Zaki AM, van Boheemen S, Bestebroer TM, Osterhaus AD, Fouchier RA Isolation of a novel coronavirus from a man with pneumonia in Saudi Arabia [published correction appears in NEngl J Med. 2013;369(4):394]. N Engl J Med. 2012;367(19):1814-1820.

3. Phelan AL, Katz R, Gostin LO. The novel coronavirus originating in Wuhan, China: Challenges for global health governance. JAMA. 2020; 323(8):709-710.

4. COVID-19 Dashboard by the Center for Systems Science and Engineering (CSSE) at Johns Hopkins University (JHU). https://coronavirus. jhu.edu/map.html. Accessed August 28, 2020.

5. Wang D, Hu B, Hu C, et al. Clinical characteristics of 138 hospitalized patients with 2019 novel coronavirus-infected pneumonia in Wuhan, China. JAMA. 2020;323(11):1061-1069.

6. Huang C, Wang Y, Li X, et al. Clinical features of patients infected with 2019 novel coronavirus in Wuhan, China [published correction appears in Lancet. 2020; 15;395(10223):496]. Lancet. 2020;395(10223): 497-506.

7. Richardson S, Hirsch JS, Narasimhan M, et al. Presenting characteristics, comorbidities, and outcomes among 5700 patients hospitalized with COVID-19 in the New York City area. JAMA. 2020;323(20): 2052-2059. doi:10.1001/jama.2020.6775

8. Armstrong RA, Kane AD, Cook TM. Outcomes from intensive care in patients with COVID-19: A systematic review and meta-analysis of observational studies. Anaesthesia. 2020;75(10):1340-1349. doi:10. 1111/anae.15201

9. Cardoso FS, Papoila AL, Machado RS, et al. Age, sex, and comorbidities predict ICU admission or mortality in cases with SARS-CoV2 infection: A population-based cohort study. Crit Care. 2020;24:465.

10. Wu Z, McGoogan JM. Characteristics of and important lessons from the coronavirus disease 2019 (COVID-19) outbreak in China: Summary of a report of 72314 cases from the Chinese Center for Disease Control and Prevention. JAMA. 2020;323(13):1239-1242.
11. Rodriguez-Morales AJ, Cardona-Ospina JA, Gutiérrez-Ocampo E, et al: Latin American Network of Coronavirus Disease 2019-COVID-19 Research (LANCOVID-19). Clinical, laboratory and imaging features of COVID-19: A systematic review and meta-analysis. Travel Med Infect Dis. 2020;34:101623.

12. Nowak B, Szymański P, Pańkowski I, et al. Clinical characteristics and short-term outcomes of patients with coronavirus disease 2019: A retrospective single-center experience of a designated hospital in Poland. Pol Arch Intern Med. 2020;130(5):407-411.

13. Yang J, Zheng Y, Gou X, et al. Prevalence of comorbidities and its effects in patients infected with SARS-CoV-2: A systematic review and meta-analysis. Int J Infect Dis. 2020;94:91-95.

14. Fosbøl EL, Butt JH, Østergaard L, et al. Association of angiotensinconverting enzyme inhibitor or angiotensin receptor blocker use with COVID-19 diagnosis and mortality. JAMA. 2020;324(2):168-177.

15. Liu Y, Mao B, Liang S, et al. Association between age and clinical characteristics and outcomes of COVID-19. Eur Respir J. 2020;55(5):2001112.

16. Yang AP, Liu JP, Tao WQ, Li HM. The diagnostic and predictive role of NLR, d-NLR and PLR in COVID-19 patients. Int Immunopharmacol. 2020;84:106504.

17. Henry BM, Aggarwal G, Wong J, et al. Lactate dehydrogenase levels predict coronavirus disease 2019 (COVID-19) severity and mortality: A pooled analysis. Am J Emerg Med. 2020;38(9):1722-1726.

18. Li Y, Zhao K, Wei H, et al. Dynamic relationship between D-dimer and COVID-19 severity. Br J Haematol. 2020. doi:10.1111/bjh.16811

19. Zhang L, Yan X, Fan Q, et al. D-dimer levels on admission to predict in-hospital mortality in patients with COVID-19. J Thromb Haemost. 2020;18(6):1324-1329.

20. Aziz M, Fatima R, Assaly R. Elevated interleukin- 6 and severe COVID-19: A meta-analysis. J Med Virol. 2020;92(11):2283-2285.

21. Wang L. C-reactive protein levels in the early stage of COVID-19. Médecine et Maladies Infectieuses. 2020;50:332-334.

22. Wendel Garcia PD, Fumeaux T, Guerci P, et al; RISC-19-ICU Investigators. Prognostic factors associated with mortality risk and disease progression in 639 critically ill patients with COVID-19 in Europe: Initial report of the international RISC-19-ICU prospective observational cohort. EClinicalMedicine. 2020;2020:100449.

23. Weinstock MB, Echenique ANA, Russell JW. Chest X-ray findings in 636 ambulatory patients with COVID-19 presenting to an urgent care center: A normal chest X-ray is no guarantee. J Urgent Care Med. 2020:5:13-18

24. Horby P, Lim WS, Emberson JR, et al; RECOVERY Collaborative Group. Dexamethasone in hospitalized patients with COVID-19: Preliminary report. N Engl J Med. 2020. doi:10.1056/NEJMoa2021436

25. Beigel JH, Tomashek KM, Dodd LE, et al; ACTT-1 Study Group Members. Remdesivir for the treatment of COVID-19: Preliminary report. N Engl J Med. 2020;383(19):1813-1826. doi:10.1056/NEJMoa2007764

26. Intensive Care National Audit \& Research Centre (ICNARC). COVID-19 Report. https://www.icnarc.org/Our-Audit/Audits/Cmp/Reports. London, UK: ICNARC; 2020. Accessed June 16, 2020. 